\title{
Analytical Estimation of Elastic Properties of Polypropylene Fiber Matrix Composite by Finite Element Analysis
}

\author{
Bhaskar Pal ${ }^{1}$, Mohamed Riyazuddin Haseebuddin ${ }^{2}$ \\ ${ }^{1}$ Mechanical Engineering Department, Dayananda Sagar Academy of Technology \\ and Management, Bangalore, India \\ ${ }^{2}$ Mechanical Engineering Department, Dayananda Sagar College of Engineering, Bangalore, India \\ Email: pbhaskar70@yahoo.com, thereckoner@rediffmail.com
}

Received November 15, 2011; revised December 24, 2011; accepted January 3, 2012

\begin{abstract}
A structural composite is a material system consisting of two or more phases on a macroscopic scale, whose mechanical performance and properties are designed to be superior to those of constituent materials acting independently. Fiber reinforced composites (FRP) are slowly emerging from the realm of advanced materials and are replacing conventional materials in a variety of applications. However, the mechanics of FRPs are complex owing to their anisotropic and heterogeneous characteristics. In this paper a representative volume model has been considered and a finite element model incorporating the necessary boundary conditions is developed using available FEA package ANSYS to predict the elastic property of the composite. For verification, the numerical results of elastic properties are compared with the analytical solution and it is found that there is a good agreement between these results.
\end{abstract}

Keywords: FRP; RVE; Finite Element Method; Homogenization

\section{Introduction}

In recent years, there has been a rapid growth in the use of fiber-reinforced composites due to their ability to replace competitive materials on the basis of lower density and equivalent strength, low thermal conductivity, high corrosion and wear resistance and the possibility of combining the toughness of thermoplastic polymers with the stiffness and strength of reinforcing fibres. This has resulted in the need for variety of application like general, structural, automotive and aerospace industry.

Unidirectional composites are those which have all fibres aligned in a single direction. A unidirectional composite with a hexagonal array of fibres can be transversely isotropic because the properties are the same along any plane which is normal to the fibre direction [1]. The stiffness and strength of a unidirectional composite are anisotropic properties since they vary with orientations. The stiffness of unidirectional composites in the fibre direction is usually dominated by the fibre properties while the strength in the transverse direction is dominated by the matrix properties. Since the strength of a unidirectional composite under transverse tension is much smaller than under longitudinal tension, transverse tensile loading is believed to be the critical loading of unidirec- tional composite materials [2].

Composite elastic properties are determined by the physical and mechanical properties of the individual materials. Some analytical and numerical techniques have been used for prediction and characterization of composite behaviour. Analytical methods provide reasonable prediction for relatively simple configurations of the phases. Complicated geometries, loading conditions and material properties often do not yield analytical solutions, due to complexity and the number of equations. In this case, numerical methods are used for approximate solutions, but they still make some simplifying assumptions about the inherent microstructures of heterogeneous multiphase materials, one such method is finite element analysis [3-5].

One of the basic requirements in the mechanics of composite materials is to determine the effective elastic properties. Experimental determination of the moduli of unidirectional composites is difficult, especially when it involves determining the longitudinal shear and transverse shear moduli. Thus, numerical techniques like the finite element method are needed to calculate these shear moduli. Numerical methods to calculate composite material properties usually involve analysis of a representative volume element (RVE) [6-8]. 
A great number of micromechanical models have been proposed in the literature for predicting various mechanical properties of composite materials [9-11]. Several other models have been proposed such as numerical homogenization [9], FEM.

Among all the theoretical models, the ROM has got the simplest mathematical relations. To apply these models, the modulus of elasticity of the polymer, $\mathrm{E}_{\mathrm{m}}$, and of the fiber, $E_{f}$, should be known and then the modulus of elasticity of the composite, $\mathrm{E}_{1,2}$, can be calculated for any volume fraction of the fiber in the composition. But those experienced in the field shall admit that this model, in most cases, do not predict the modulus of elasticity of the composites satisfactorily. The experimental observations and analysis also confirm that [5,12-14].

There are a few issues that need to be verified carefully when carrying out such analyses. Firstly, the correct RVE corresponding to the assumed fibre distribution must be isolated. Secondly, correct boundary conditions need to be applied to the chosen RVE to model different loading situations. Proper consideration must be given to the periodicity and symmetry of the model in arriving at the correct boundary condition.

In the present work, the procedure for predicting the elastic constants of the composite from the RVE is established for a micromechanical three-dimensional finite element analysis. The finite element calculations are made at a specific volume fraction because the geometry of the regions of the finite-element mesh that represent the fibre and matrix differ from one volume fraction to the next and, therefore, each volume fraction study requires a separate analysis. The finite element method is adopted for predicting various elastic properties of unidirectional oriented FRP and the results of $E_{1}, E_{2}, v_{12}$ and $\mathrm{V}_{23}$ are compared with the rule of mixtures and HalphinTsai criteria.

\section{Methodology}

\subsection{Analytical Formulation}

\subsubsection{Role of Mixture}

Rules of Mixtures are mathematical expressions which give some property of the composite in terms of the properties, quantity and arrangement of its constituents. The notations will be established by use of the following relationships:

- Longitudinal Young's Modulus

$$
\mathrm{E}_{1}=\mathrm{E}_{\mathrm{f}} \mathrm{V}_{\mathrm{f}}+\mathrm{E}_{\mathrm{m}}\left(1-\mathrm{V}_{\mathrm{f}}\right)
$$

- Transverse Young's Modulus

$$
\frac{1}{E_{2}}=\frac{V_{f}}{E_{f}}+\frac{\left(1-V_{f}\right)}{E_{m}}
$$

- Major Poisson's Ratio

$$
\mathrm{v}_{12}=\mathrm{v}_{\mathrm{f}} \mathrm{V}_{\mathrm{f}}+\mathrm{v}_{\mathrm{m}}\left(1-\mathrm{V}_{\mathrm{f}}\right)
$$

- In-Plane Shear Modulus

$$
\frac{1}{G_{12}}=\frac{V_{f}}{G_{f}}+\frac{\left(1-V_{f}\right)}{G_{m}}
$$

\subsubsection{Semi-Empirical Model (Halphin-Tsai)}

Halphin and Tsai developed their models as simple equations by curve fitting to results that are based on elasticity. The equations are semi-empirical in nature since involved parameters in the curve fitting carry physical meaning.

- Longitudinal Young's Modulus

$$
\mathrm{E}_{1}=\mathrm{E}_{\mathrm{f}} \mathrm{V}_{\mathrm{f}}+\mathrm{E}_{\mathrm{m}} \mathrm{V}_{\mathrm{m}}
$$

- Transverse Young's Modulus

$$
\frac{\mathrm{E}_{2}}{\mathrm{E}_{\mathrm{m}}}=\left[\frac{1+\xi \eta \mathrm{V}_{\mathrm{f}}}{1-\eta \mathrm{V}_{\mathrm{f}}}\right], \eta=\left[\frac{\left[\frac{\mathrm{E}_{\mathrm{f}}}{\mathrm{E}_{\mathrm{m}}}\right]-1}{\left[\frac{\mathrm{E}_{\mathrm{f}}}{\mathrm{E}_{\mathrm{m}}}\right]+\xi}\right]
$$

The term " $\xi$ " is called the reinforcing factor and depends on the following;

Fiber geometry, Packing geometry, Loading condition

- Major Poisson's Ratio

$$
\mathrm{v}_{12}=\mathrm{v}_{\mathrm{f}} \mathrm{V}_{\mathrm{f}}+\mathrm{v}_{\mathrm{m}} \mathrm{V}_{\mathrm{m}}
$$

- In-Plane Shear Modulus

$$
\frac{\mathrm{G}_{12}}{\mathrm{G}_{\mathrm{m}}}=\left[\frac{1+\xi \eta \mathrm{V}_{\mathrm{f}}}{1-\eta \mathrm{V}_{\mathrm{f}}}\right], \eta=\left[\frac{\left[\frac{\mathrm{G}_{\mathrm{f}}}{\mathrm{G}_{\mathrm{m}}}\right]-1}{\left[\frac{\mathrm{G}_{\mathrm{f}}}{\mathrm{G}_{\mathrm{m}}}\right]+\xi}\right]
$$

\subsection{Compliance Matrix}

In composite material fibers may be oriented in an arbitrary manner. Depending on the arrangements of the fibers, the material may behave differently in different directions. According to their behaviour, composites may be characterized as generally anisotropic, monoclinic, orthotropic, and transversely isotropic. In this paper, transversely isotropic characteristics have been considered for the fiber reinforced composite and fiber arrangement as shown in the Figure 1.

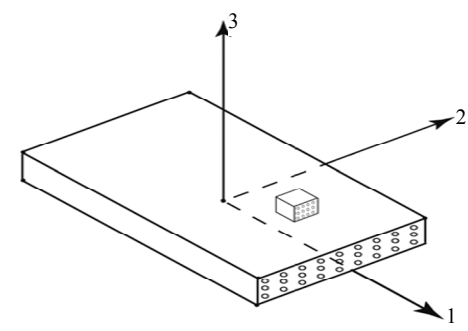

Figure 1. Arrangement of fiber direction for transversely isotropic composite. 


$$
\left[\begin{array}{c}
\epsilon_{\mathrm{x}} \\
\epsilon_{\mathrm{y}} \\
\epsilon_{\mathrm{z}} \\
\gamma_{23} \\
\gamma_{13} \\
\gamma_{12}
\end{array}\right]=\left[\begin{array}{ccc}
1 / \mathrm{E}_{1} & -\gamma_{12} / \mathrm{E}_{2} & -\gamma_{12} / \mathrm{E}_{2} \\
-\gamma_{12} / \mathrm{E}_{1} & 1 / \mathrm{E}_{2} & -\gamma_{23} / \mathrm{E}_{2} \\
-\gamma_{12} / \mathrm{E}_{1} & -\gamma_{23} / \mathrm{E}_{2} & 1 / \mathrm{E}_{2} \\
0 & 0 & 0 \\
0 & 0 & 0 \\
0 & 0 & 0
\end{array}\right.
$$

Here 1-2-3 orthogonal coordinate system is used where the directions are taken as follows:

- The 1-axis is aligned with the fiber direction.

- The 2-axis is in the plane of the layer and perpendicular to the fibers.

- The 3-axis is perpendicular to the plane of the layer and thus also perpendicular to the fibers.

Stress strain relationship for compliance matrix for transversely isotropic matrix given below.

\subsection{Representative Volume Element (RVE)}

Primary to use of numerical approximations of the effective properties of composite is the concept of representative volume element (RVE). Square or cubic RVEs are used for most numerical approximations because of the ease of numerically solving boundary values problems with these geometries. The difficulties involved in generating statistical information about particle distributions and concentrations leads to difficulties in the rigorous determination of RVE sizes. Hence, for most applications, RVE sizes have been rather arbitrary. In this paper a RVE model of $420 \mu \mathrm{m} \times 420 \mu \mathrm{m}$ has considered which consists of different volume fraction of fiber density. In the model calculations, cuboid will be matrix and cylinder will be fiber. Figure 2 shows the typical RVE which consists of $10 \%, 17 \%, 27 \%, 40 \%$ and $50 \%$ of fiber content.

\subsection{Homoginization}

In classical lamination theory the composite lamina is modelled as a homogeneous orthotropic medium with certain effective moduli that describe the "average" material properties of the composite. To describe this macroscopically homogeneous medium, macro-stress and macro-strain are derived by averaging the stress and strain tensor over the volume of the RVE. The average stress and strain quantities defined in Equations (9) and (10) thus ensure equivalence in strain energy between the equivalent homogeneous material and the original heterogeneous material. These average quantities will be used in the subsequent analysis to determine composite moduli

$$
\overline{\sigma_{i j}}=\frac{1}{\mathrm{~V}} \int_{\mathrm{v}} \sigma_{i j} \cdot \mathrm{dV}
$$

$$
\left.\begin{array}{ccc}
0 & 0 & 0 \\
0 & 0 & 0 \\
0 & 0 & 0 \\
2\left(1+\gamma_{23}\right) / \mathrm{E}_{2} & 0 & 0 \\
0 & 1 / \mathrm{G}_{12} & 0 \\
0 & 0 & 1 / \mathrm{G}_{12}
\end{array}\right] \times\left[\begin{array}{c}
\sigma_{x} \\
\sigma_{y} \\
\sigma_{z} \\
\tau_{23} \\
\tau_{13} \\
\tau_{12}
\end{array}\right]
$$

\section{Computational Details}

In this present work, finite element method is used to approximate the different elastic property of the fiber reinforced composites by ANSYS 12.

Assumptions made for the present analysis were

- The composite is

- Macroscopically homogeneous;

- Linearly elastic;

- Macroscopically transversely isotropic;

- Initially stress free (no thermal stress).

- The fibers are:

- Homogeneous;

- Linearly elastic;

- Isotropic;

- Regularly spaced;

- Perfectly aligned.

- The matrix is:

- Homogeneous;

- Linearly elastic;

• Isotropic.

\subsection{Modelling}

A regular three-dimensional arrangement of short fibre in a matrix was adequate to describe the overall behaviour of the composite, was modelled as a regular uniform arrangement, as shown in Figure 1. This model assumed that the fibre was a perfect cylinder of length $250 \mu \mathrm{m}$, and diameter $(\mathrm{d}=50 \mu \mathrm{m})$ in a cube $(420 \times 420 \times 250$ $\mu \mathrm{m}^{3}$ ) of matrix. It is assumed that the geometry, material and loading of the unit cell are symmetrical with respect to $x-y-z$ coordinate system as shown in Figure 2. Therefore, $10 \%$ volume fraction i.e. 9 fibers has been inserted in a cubic matrix $\left(420 \times 420 \times 250 \mu^{3}\right)$ uniformly as shown in Figure 2. 3D finite element meshing for the $10 \%$ volume fraction of the fiber has been done, similarly the finite element modelling and meshing were done by varying the fiber volume fraction from $10 \%$ to $54 \%$ and as shown in Figure 3.

\subsection{Element Type}

The element SOLID 45 of ANSYS V12.0 used for the 


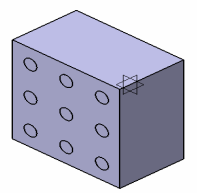

$10 \%$

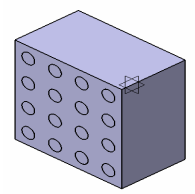

$17 \%$

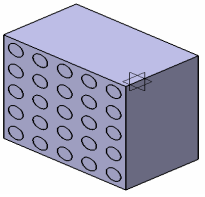

$27 \%$

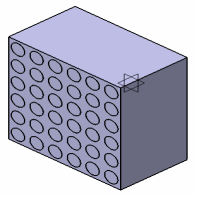

$40 \%$

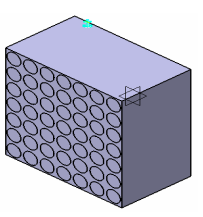

$54 \%$

Figure 2. Unit cell of square array fibre packing geometry for RVE model.

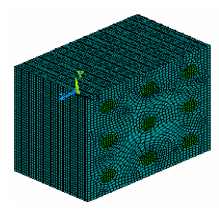

$10 \%$

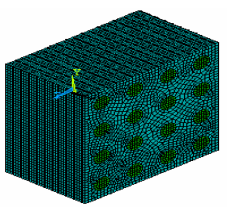

$17 \%$

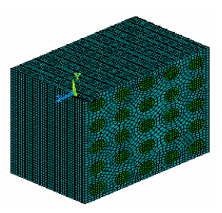

$27 \%$

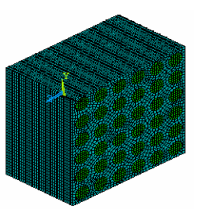

$40 \%$

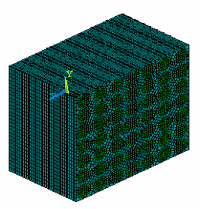

$54 \%$

Figure 3. Finite element mesh for $\mathbf{1 0} \%$ volume fraction.

present analysis which is based on a general 3D state of stress and is suited for modeling 3D solid structure under 3D loading. The element has 8 noded brick element with three degrees of freedom per node (UX, UY and UZ).

\subsection{Boundary Condition}

In this work the boundary condition with normal strain applied in $\mathrm{x}$ direction are as follows [11]:

$$
\mathrm{u}(\mathrm{LF})=0, \mathrm{v}(\mathrm{BF})=0, \mathrm{w}(\mathrm{BKF})=0 \text { and } \mathrm{u}(\mathrm{RF})=\delta
$$

where LF, BF, BKF and RF stand for left face, bottom face, back face and right face of the RVE model. All other faces are free of any displacement.

In this paper, axial loading is modelled by a displacement acting on the plane yz at $\mathrm{x}$. For such loading conditions, the boundaries of the RVE also correspond to lines of symmetry. Thus, normal displacements of the boundaries of the quadrant are restricted to those that cause the boundary to displace only parallel to the original boundary.

The displacement constraints applied to the finite element model to determine $\mathrm{E}_{1}$ are as follows and as shown in Figure 4.

$$
\begin{aligned}
& \mathrm{u}_{\mathrm{x}}(0, \mathrm{y}, \mathrm{z})=0 \\
& \mathrm{u}_{\mathrm{x}}(\mathrm{x}, \mathrm{y}, \mathrm{z})=\delta_{\mathrm{x}}=5.0 \mathrm{E}-05 \\
& \mathrm{u}_{\mathrm{y}}(\mathrm{x}, 0, \mathrm{z})=0 \\
& \mathrm{u}_{\mathrm{y}}(\mathrm{x}, \mathrm{y}, \mathrm{z})=\text { constant }=\delta_{\mathrm{y}} \\
& \mathrm{u}_{\mathrm{z}}(\mathrm{x}, \mathrm{y}, 0)=0 \\
& \mathrm{u}_{\mathrm{z}}(\mathrm{x}, \mathrm{y}, \mathrm{z})=\text { constant }=\delta_{\mathrm{z}}
\end{aligned}
$$

\subsection{Material Property}

The different material properties of both matrix and fiber has shown in the Table 1.

Material properties are used to determine the several elastic properties of the composite that is Longitudinal young's modulus $\left(E_{1}\right)$ and Transverse young's modulus $\left(E_{2}\right)$ by varying the volume fraction of the fiber.

\subsection{Sample Analysis}

Figure 5 shows the analysis of $40 \%$ of volume fraction of fiber reinforced composite in which the von misses stresses for the matrix fiber and composite has been indicated. The stresses are obtained by applying the displacement loading in longitudinal direction.

\section{Results and Discussion}

\subsection{Longitudinal Young's Modulus $\left(E_{1}\right)$}

Figure 6 shows comparison of finite element data, rule of mixtures and Halphin-Tasi results for composite modulli $E_{1}$ at different volume fractions. The finite-element solution gives identical results to the rule of mixtures and semi empirical analytical formulation. The linear dependence of E1 on fibre volume fraction is demonstrated and, as expected, the modulus increases while increasing the fibre volume fraction.

\subsection{Transverse Young's Modulus $\left(E_{2}\right)$}

Figure 7 shows comparison of finite element data, rule of mixtures and Halphin-Tasi results for composite modulli $E_{2}$ at different volume fractions. Finite element results are as expected; on the assumption that the composite is macroscopically transversely isotropic the values obtained for the whole unit cells investigated are perfectly coincident. The Rule of mixtures model, the Halpin-Tsai equation and data from the finite-element calculations are compared. The term " $\xi$ " is called the reinforcing factor in Halpin Tsai equation and depends on the fiber geometry, packing geometry, loading condition. As the fibers are circular and packing density is increasing 

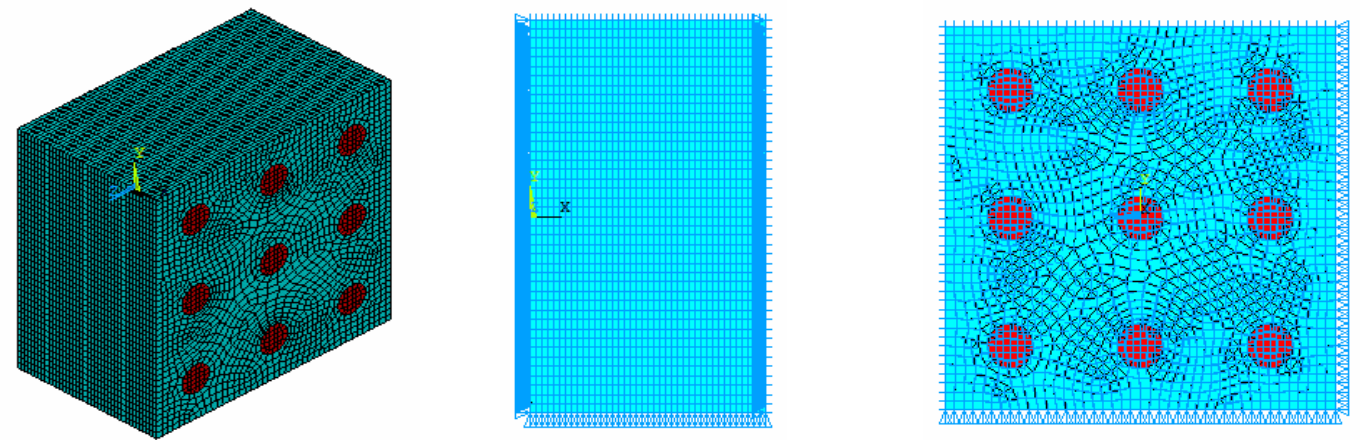

Figure 4. Boundary condition for longitudinal modulus of composite in $\mathrm{x}$ direction.

Table 1. Shows the material properties of composites.

\begin{tabular}{ccc}
\hline Material Properties & Polypropylene co ethylene (PPE) & Polypropylene (PP) \\
\hline Longitudinal Modulus E 1 & $1.05 \mathrm{Gpa}$ & $4.5 \mathrm{Gpa}$ \\
Transverse Modulus E 2 & $1.05 \mathrm{Gpa}$ & $4.5 \mathrm{Gpa}$ \\
Poisson's Ratio & 0.33 & 0.2 \\
\hline
\end{tabular}
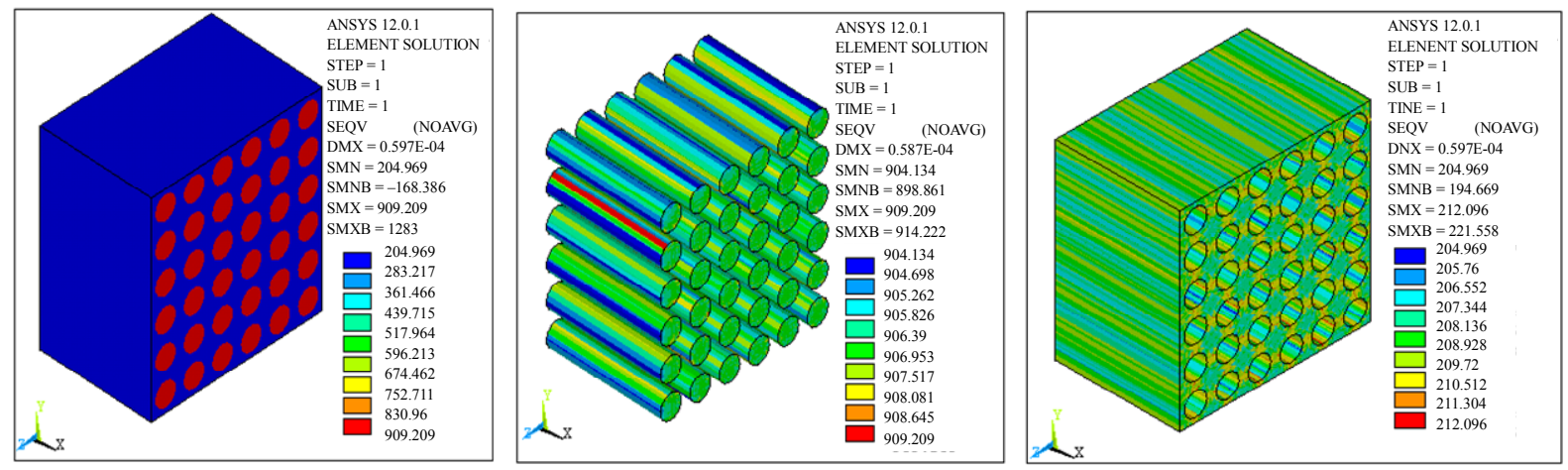

Figure 5. Stress distribution of matrix, fiber and composite during computing of $E_{1}$.

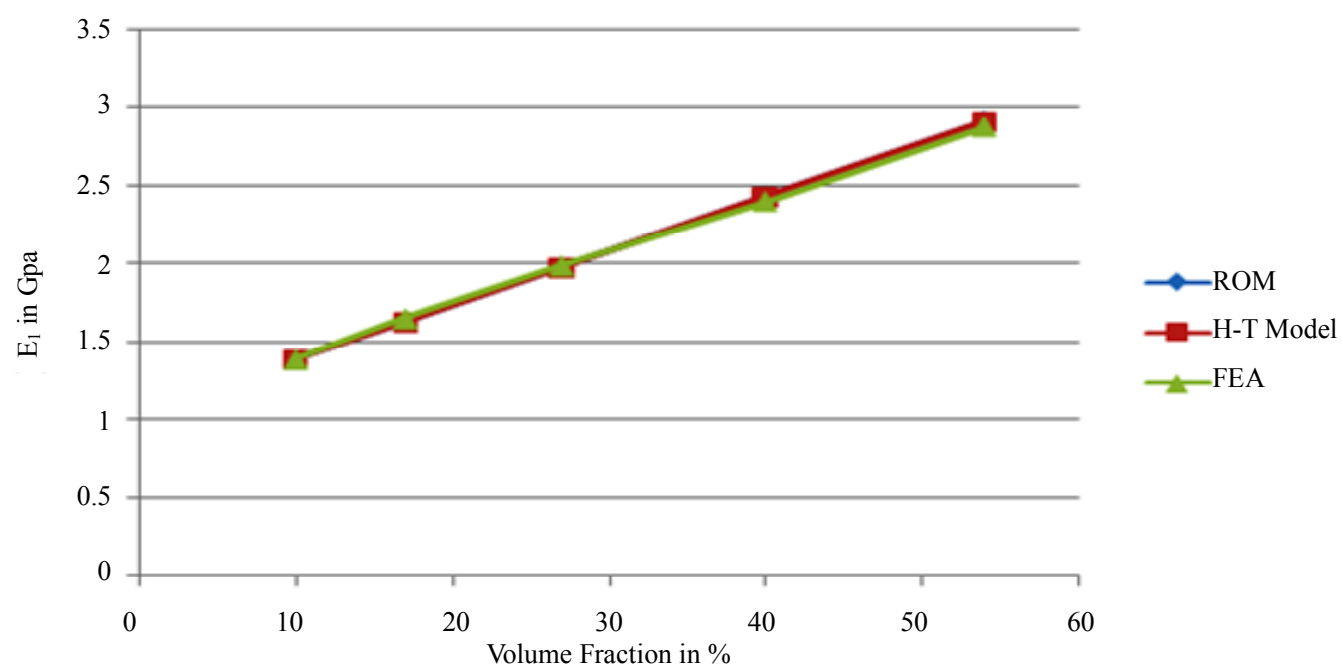

Figure 6. Comparison of finite element data, rule of mixtures and Halphin-Tasi results for composite modulli $\mathbf{E}_{1}$ for different volume fration of fiber. 


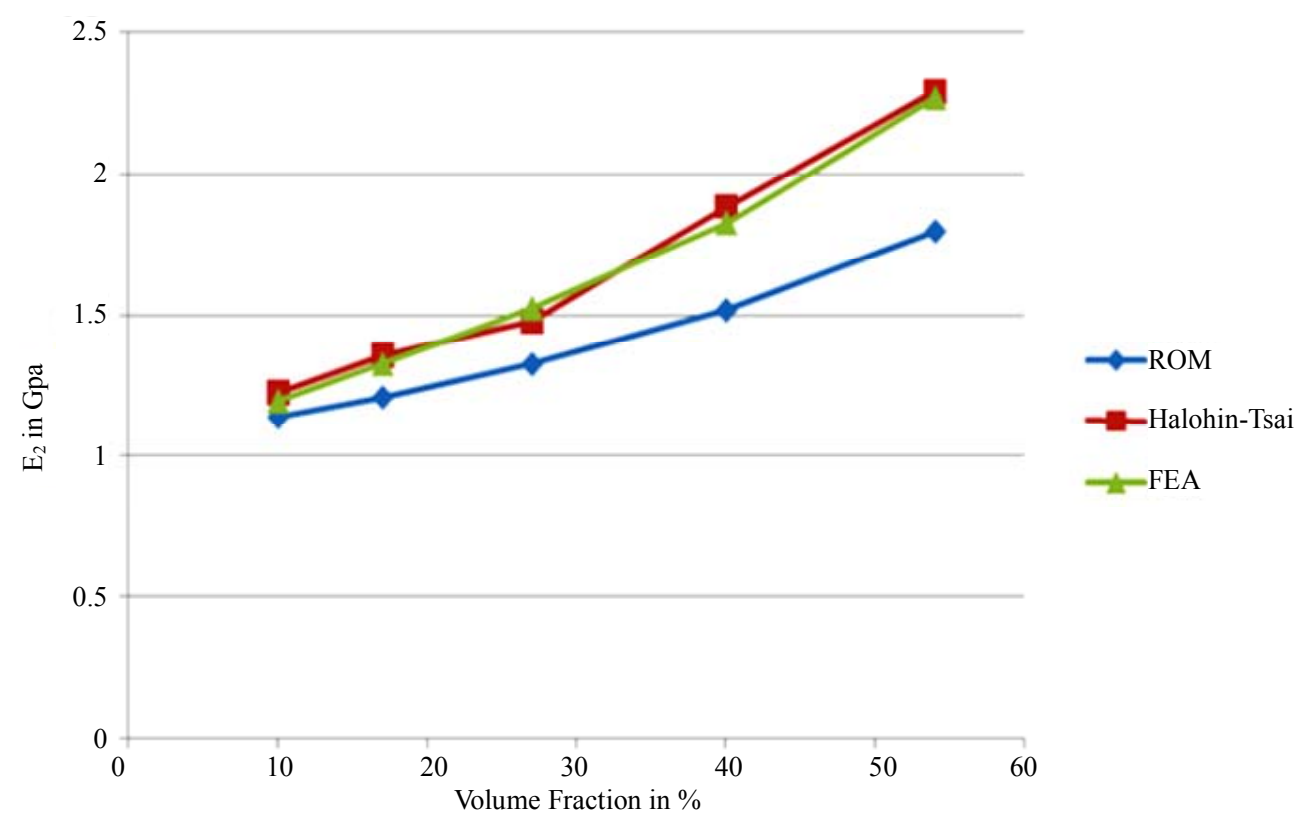

Figure 7. Comparison of finite element data, rule of mixtures and Halphin-Tasi results for composite modulli $E_{2}$ for different volume fration of fiber.

accordingly the " $\xi=2$ " is adapted to consider those changes in the composites. In this Halpin-Tsai equation shows a good agreement between finite element values. But in Rule of mixture there is no such parameter which can be considered for those changes. Due to this reason The Rule of mixture is not showing the good agreement with finite element data.

\subsection{Poisson's Ratio}

The major Poisson's ratio $\vartheta_{12}$ for the composite is defined as minus the ratio of strain in the y-direction divided by the strain in the $\mathrm{x}$-direction when only the stress $\sigma_{x}$ is applied. The Rule of Mixtures expression for the composite Poisson's ratio is similar in form to the expression for $E_{1}$. It evident from the Figure 8 that analytical and numerical results are in perfect agreement although data from the Rules of Mixtures are slightly higher than the finite-element results.

The Poisson's ratio $\vartheta_{23}$ describes the contraction in the z-direction on $\vartheta_{23}$ applying loads in the y-direction. No analytical formulas have been applied to compare with numerical results of Poisson's ratio. It is worthwhile to show finite element results of both with different fibre volume fractions. As expected from the Figure 9, $\vartheta_{23}$ appears to be higher than $\vartheta_{12}$ and the comparison between the two Poisson's ratios shows that Poisson's ratios $\vartheta_{12}$ are less sensitive to the fibre volume fraction. In particular, the values of $\vartheta_{12}$ are quite close to the Poisson's ratio of polypropylene (PP) especially at higher fibre volume fraction. On the contrary, $\vartheta_{23}$ is strongly dependent from the fibre volume fraction and, its values approaches the Poisson's ratio of the Polypropylene co ethylene at lower values of the fibre volume fraction.

\section{Conclusions}

1) The $3 \mathrm{D}$ unit cell of the unidirectional fiber reinforced composite has been identified (RVE) as a basic building block for estimating overall mechanical composite properties.

2) Various micromechanical methods to determine the elastic behaviour of composite materials have been discussed.

3) Finite element analysis has provided an implicit means of modelling polymer composites.

4) Numerical homogenization tools have been developed for the evaluation of the effective material properties of the short fiber composites.

5) The appropriate constraints on the RVE under various loadings have been determined from symmetry boundary conditions, obtaining a nearly complete set of elastic constants for a three-dimensional unidirectional composite.

6) The results of elastic moduli $\mathrm{E}_{1}, \mathrm{E}_{2}, \mathrm{E}_{2}, v_{12}, v_{23}$, compared with the results of analytical solution and it is found that the results from FE simulation are in good agreement with the analytical results employed in this exercise namely the Halpin-Tsai semi-empirical expression and to some extent the Rules of Mixtures.

7) As this mechanical property of fiber-filled composites are affected by a number of parameters such as fiber type, matrix type, fiber orientation, fiber geometry, volume fraction of the fibers and the degree of interfacial 


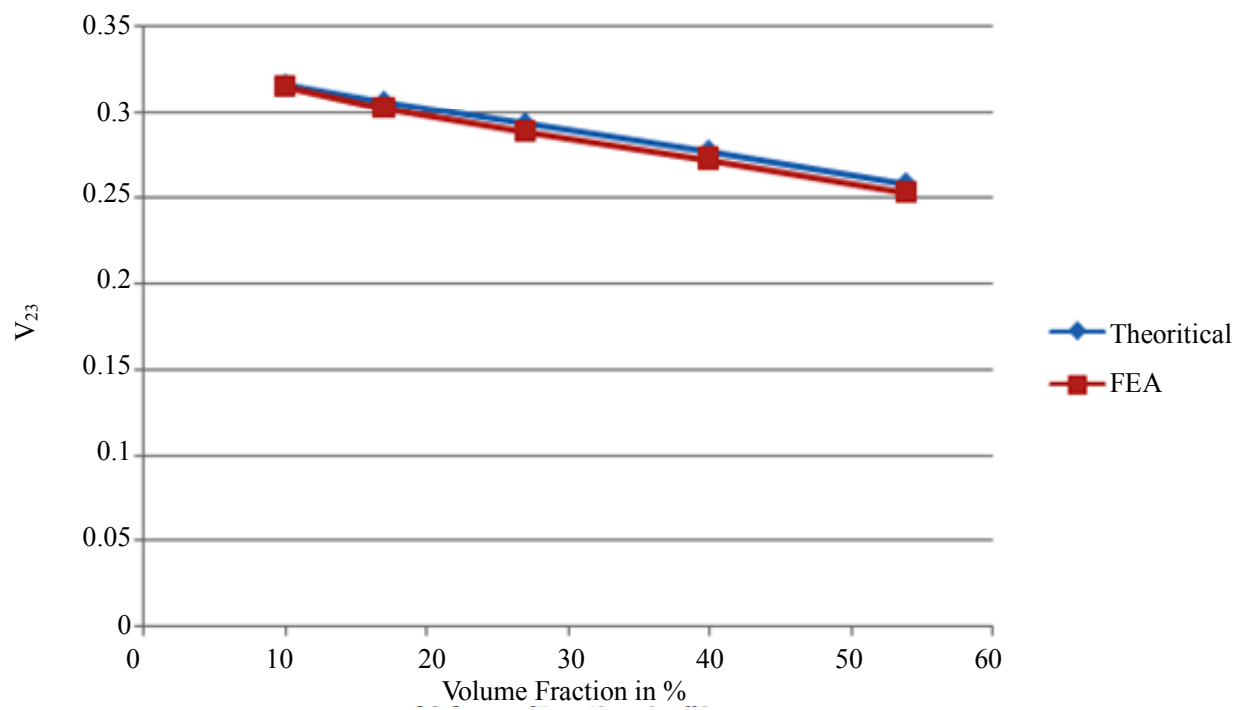

Figure 8. Comparison of finite element data and rule of mixtures results for composite poisson's ratio $\vartheta_{12}$.

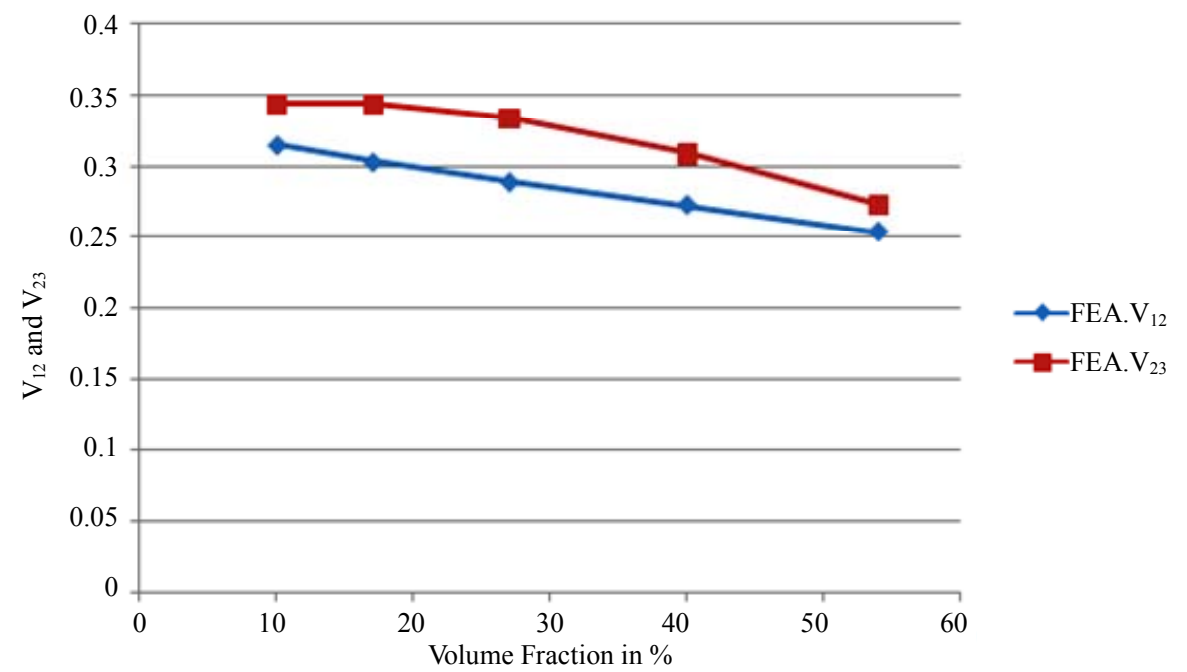

Figure 9. Comparison of finite element results for composite poisson's ratio $\vartheta_{12}$ and $\vartheta_{23}$.

adhesion between the fiber and the polymer matrix, the ROM has not been adopted by this property expect the volume fraction. So the results are also not so accurate.

8) The Halpin-Tsai (HT) model is also a theoretical model. This model, besides the modulus of elasticity of the polymer, $\mathrm{E}_{\mathrm{m}}$, and of the fiber, $\mathrm{E}_{\mathrm{f}}$, includes a geometrical parameter (aspect ratio) of the fiber as well. The model has a complicated mathematical structure with fiber geometry, packing geometry, loading condition. This factors help to showing the good agreement with finite element data.

9) It is observed that the change in volume fraction of fiber has a significant effect on elastic properties.

\section{REFERENCES}

[1] D. F. Adams and D. R. Doner, "Longitudinal Shear Load- ing of a Unidirectional Composite," Journal Composite Materials, Vol. 1, No. 1, 1967, pp. 4-17.

[2] D. F. Adams and D. R. Doner, "Transverse Normal Loading of a Unidirectional Composite," Journal Composite Materials, Vol. 1, No. 2, 1967, pp. 152-164. doi: $10.1177 / 002199836700100205$

[3] S. Houshyar, R. A. Shanks and A. Hodzic, "Modelling of Polypropylene Fibre-Matrix Composites Using Finite Element Analysis," Express Polymer Letters, Vol. 13, No. 1, 2009, pp. 2-12.

[4] S. Houshyar, R. A. Shanks and A. Hodzic, "The Effect of Fibre Concentration on Mechanical and Thermal Properties of Fibre Reinforced Polypropylene Composites," Journal of Applied Polymer Science, Vol. 96, No. 6, 2005, pp. 2260-2272. doi:10.1002/app.20874

[5] G. Kalaprasad, K. Joseph and S. Thomas, "Theoretical Modelling of Tensile Properties of Short Sisal Fibre-Reinforced Low Density Polyethylene Composites," Journal 
of Materials Science, Vol. 32, No. 16, 1999, pp. 42614267. doi:10.1023/A:1018651218515

[6] C. T. Sun and R. S. Vaidya, "Prediction of Composite Properties from a Representative Volume Element," Composites Science and Technology, Vol. 56, No. 2, 1996, pp. 171-179. doi:10.1016/0266-3538(95)00141-7

[7] S. Li, "General Unit Cell for Micromechanical Analyses of Unidirectional Composites," Composites Part A, Vol. 32, No. 6, 2000, pp. 815-816. doi:10.1016/S1359-835X(00)00182-2

[8] B. R. Kim and H. K. Lee, "An RVE-Based Micromechanical Analysis of Fiber-Reinforced Composites Considering Fiber Size Dependency," Composite Structures, Vol. 90, No. 4, 2009, pp. 418-427. doi:10.1016/j.compstruct.2009.04.025

[9] P. De Buhan and A. Taliercio, "A Homogenisation Approach to the Yield Strength of Compositematerials," European Journal of Mechanics, Vol. 10, No. 2, 1991, pp. 129-154.

[10] Y. Pan, L. Iorga and A. A. Pelegri, "Numerical Generation of a Random Chopped Fiber Composite RVE and Its Elastic Pproperties," Composites Science and Technology,
Vol. 68, No. 13, 2008, pp. 2792-2798. doi:10.1016/j.compscitech.2008.06.007

[11] M. Porfiri and N. Gupta, "Effect of Volume Fraction and Wall Thickness on the Elastic Properties of Hollow Particle Filled Composites," Composites Part B: Engineering, Vol. 40, No. 2, 2009, pp. 166-173.

doi:10.1016/j.compositesb.2008.09.002

[12] K. Hbaieb, Q. Wang, Y. H. J. Chia and B. Cotterell, "Modelling Stiffness of Polymer/Clay Nanocomposites," $\mathrm{Po}$ lymer, Vol. 48, No. 3, 2007, pp. 901-909. doi:10.1016/j.polymer.2006.11.062

[13] A. G. Facca, M. T. Kortschot and N. Yan, "Predicting the Elastic Modulus of Natural Fibre Reinforced Thermoplastics," Composites Part A: Applied Science and Manufacturing, Vol. 37, No. 10, 2006, pp. 1660-1671. doi:10.1016/j.compositesa.2005.10.006

[14] L. Peponi, J. Biagiotti, J. M. Kenny and I. Mondragon, "Statistical Analysis of the Mechanical Properties of Natural Fibers and Their Composite Materials. II. Composite Materials," Polymer Composites, Vol. 29, No. 3, 2008, pp. 321-325. doi:10.1002/pc.20386 